\title{
A Review of GUMBOS: Tunable Ionic Materials
}

ISSN: 2576-8840

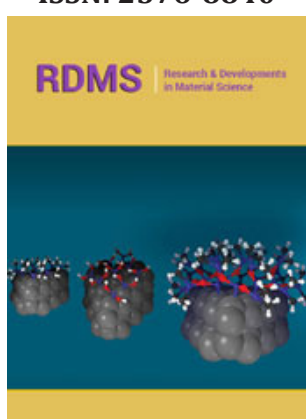

*Corresponding author: Caitlan E Ayala, Department of Chemistry, Louisiana State University, USA

Submission: 海 August 06, 2021

Published: 㘹August 16, 2021

Volume 15 - Issue 4

How to cite this article: Caitlan E Ayala, Isiah M Warner. A Review of GUMBOS Tunable Ionic Materials. Res Dev Material Sci. 15(4). RDMS.000868. 2021. DOI: 10.31031/RDMS.2021.15.000868

Copyright@ Caitlan E Ayala. This article is distributed under the terms of the Creative Commons Attribution 4.0 International License, which permits unrestricted use and redistribution provided that the original author and source are credited.

\author{
Caitlan E Ayala* and Isiah M Warner \\ Department of Chemistry, Louisiana State University, USA
}

\begin{abstract}
Strategic development of ionic materials has long been of considerable interest to many researchers. For example, ionic liquid research has been extensively explored as alternative solvents to highly volatile and/or caustic traditional organic solvents. While these solvents have been classified as having a range of melting points (below $100{ }^{\circ} \mathrm{C}$ ), many researchers have targeted relatively low melting point materials for their desired applications. Similar materials with higher melting points were often deemed inefficient and often discarded and declared useless. However, several groups have begun to explore the advantages of solid-phase ionic materials, known as a groups of uniform materials based on organic salts (GUMBOS). Many advantages arise through implementation of these solid-state ILs analogues in different fields of research. These advantages include 1) maintaining ease of synthesis using IL chemistry, 2) exploring easy tunability of solid-state materials, and 3) providing researchers with ability to easily develop materials on the nanoscale. Herein, we provide an overview of advantages of using GUMBOS for materials research applications.
\end{abstract}

Keywords: GUMBOS; NanoGUMBOS; Tunable properties

\section{Introduction}

One of the primary driving forces for development and study of ionic materials is to investigate their use as environmentally friendly solvents with relatively low melting points and low volatility. Thus, use of these solvents reduces the level of volatile organic compounds in the atmosphere [1,2]. ILs are typically composed of an organic cation and an inorganic or organic anion with melting points at or below $100{ }^{\circ} \mathrm{C}[1,3]$. Their ease of synthesis and subsequent tunable properties, such as melting point, electrical conductivity, and hydrophobicity, make them attractive solvents for dissolution of many materials. Traditionally, tunability is exploited through counterion exchange to disrupt ionic packing (Figure 1a). Subcategories of these materials are divided between those that are present in the liquid state at room temperature and frozen ILs that are solid between room temperature and an arbitrary upper temperature limit of $100{ }^{\circ} \mathrm{C}$ (frozen ILs). In a traditional biphasic reaction, hydrophobic ions are dissolved in a water immiscible solvent, and the more hydrophilic ion is stirred in water. This reaction is often performed via stirring for 24-48 hours, and the hydrophobic product is isolated by drying and washing byproduct from the organic layer. Solid salts products were often disregarded as undesired materials for dissolution purposes $[1,3]$. However, more recent research, particularly from our group, has targeted investigations into the utility of applying such solid-state ionic materials for applications in many scientific disciplines.

Our research group has incorporated these frozen IL analogues into a separate analogous class of materials defined as groups of uniform materials based on organic salts (GUMBOS). In this new classification, GUMBOS have an extended melting point range between 25 and 250 ${ }^{\circ} \mathrm{C}$. GUMBOS also maintain the strategic feature of tunable properties through ion exchange reactions to provide advantageous materials using efficient and rapid chemistry [1,3]. For example, using an ionic precursor molecule, a simple, biphasic ion-exchange reaction is often sufficient to provide target materials from ionic precursors, examples of which are displayed in Figure 1b; [4]. 
a)

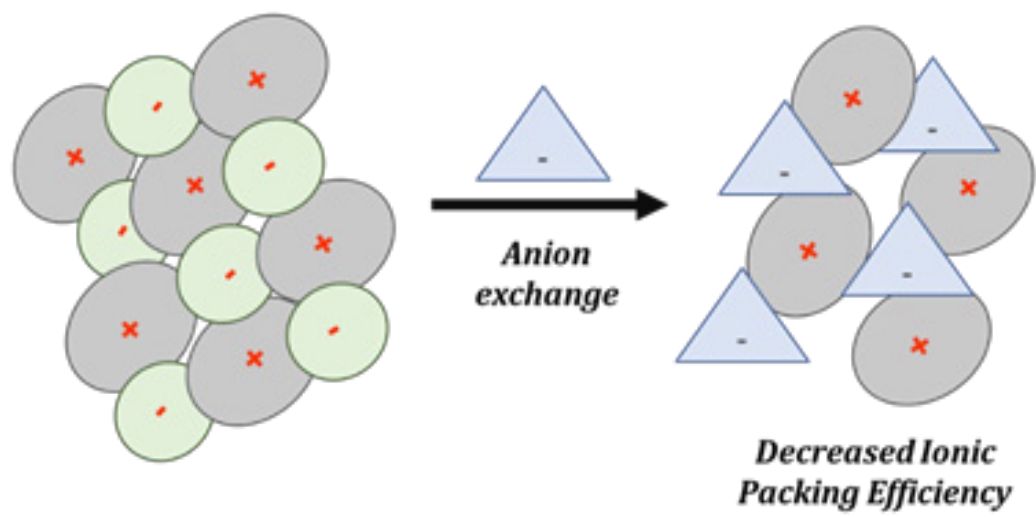

b)
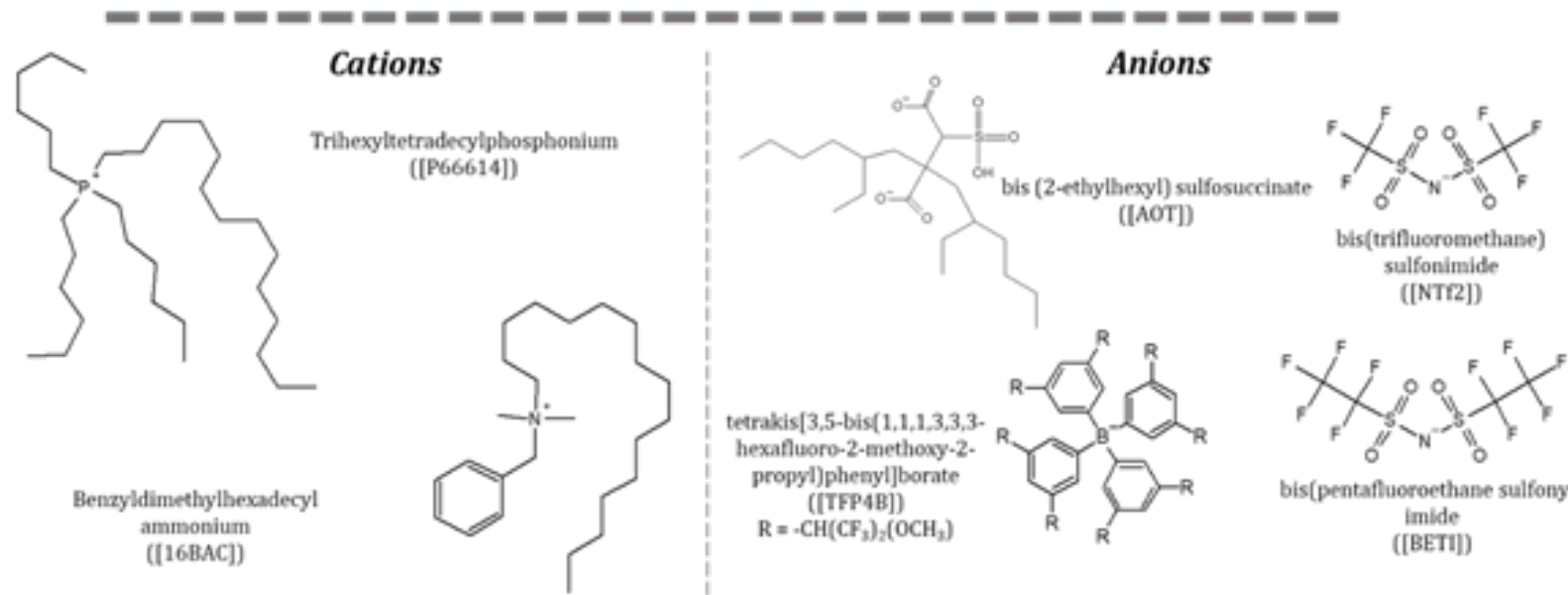

bis(pentafluoroethane sulfonyl)

imide

([BETt])

Figure 1: Schematic of a) representative ion exchange effects on packing and b) examples of common ionic materials employed in GUMBOS synthesis to increase hydrophobicity.

As a result of the numerous combinations of cations and anions, many scientific researchers have highlighted the wide variety and practical utility of solid-phase ionic materials as functional agents for sensing [5,6], optoelectronics [4,7], and development of potential antimicrobial and anticancer agents [8-12]. Extension of the melting point range for GUMBOS also provides investigators with an opportunity for operationally simple templated and nontemplated uniform nano- and micro-particle formation in aqueous systems. In such instances, relatively high concentrations of GUMBOS are typically loaded into aqueous systems and solid particles are formed via simple reprecipitation into aqueous solutions. In addition, these nano- and micro-GUMBOS particles may be tuned using a variety of parameters, one of which is counterion exchange. In many reports, this counterion tuning has been shown to provide a variety of size and stability parameters [3]. This review briefly highlights the various development strategies that can be derived through counterion exchange. Potential utility of targeted GUMBOS and nanoGUMBOS are also briefly discussed.

\section{Increasing hydrophobicity via cation exchange}

Selective tunability of hydrophobicity and material composition has led to development of many sensors for a variety of applications [6,7]. In addition, chemometric or multivariate analyses are frequently used to complement and analyze sensor responses to evaluate detection and discrimination accuracies. One application is for detection and discrimination of closely related chemical compounds such as Volatile Organic Compounds (VOCs). In one related study, Galpothdeniya and coworkers used a cation tuning strategy with [P66614] cation to increase the hydrophobicity of a $\mathrm{pH}$ indicator, bromothymol blue ([BTB]). This work demonstrated the utility of increasing and systematically varying the hydrophobicity of commercially available BTB for colorimetric and highly accurate detection and discrimination of several closely related alcoholic solvents using principal component analysis [5]. Galpothdeniya and coworkers also employed this [P66614] cation to increase the hydrophobicity of twelve different $\mathrm{pH}$ indicator dyes for use as optical sensors [5]. These researchers employed solid-state supports to create an optoelectronic chemosensor array and studied two different statistical routes for detection and discrimination of VOCs from cigarette smoke. Resultant implications of ILs as solid-state sensors were highlighted using colorimetric IL-supports with minimal dye leakage. More recently, Pramanik and coworkers have employed counterion exchange with [P66614] to synthesize methyl orange-based GUMBOS ([P66614] [MO]) as an indicator for acids and bases in organic solvents [13]. These investigators also studied the acid dissociation constants of several acids in acetonitrile. In aqueous systems, nanoGUMBOS afforded better sensory capabilities for acids and bases in water when compared to bulk forms. 
Another route for VOC detection through hydrophobic tuning has been explored through development of GUMBOS as sensory coatings for quart crystal resonators [6,14]. In order to investigate increases in hydrophobicity in the solid state, copper(II) pthalocyanine and copper(II) porphyrin underwent ion exchange with the hydrophobic [P66614] cation to form [P66614][CuTCPP] and [P66614][CuPcS4], respectively. Traditional synthetic schemes for sensory coatings often involves covalent alteration of the cyclic structures to increase hydrophobicity. When compared to traditional hydrophobic IL ([P66614][NTf2]), GUMBOS sensors produced better sensory and cross-reactive responses for eleven different VOCs. In another example of hydrophobic tuning of polycyclic structures, Kolic and coworkers studied cation exchange using a trihexyltetradecylphosphonium ([P66614]) for investigating enhancement in photosensitizing properties of porphyrins, specifically meso-tetra(4-carboxyphenyl)porphine ([TCPP]) and zinc(II) meso-tetra(4-carboxyphenyl)porphine ([ZnTCPP]) in GUMBOS and nanoGUMBOS forms [15]. These investigators determined that surface charges of nanoparticles along with optical properties and particle size were tunable through adjustment of the molar ratio of anion to cation during synthesis. It was also determined that reduction and oxidation potentials of these GUMBOS were comparable to parent compounds and that they could be employed for photosensitizing applications in DyeSensitized Solar Cells (DSSCs).

In 2020, Pramanik [16] and coworkers synthesized GUMBOS, and subsequently nanoGUMBOS, using a pyrene butyrate anion and the $\left[\mathrm{P}_{66614}\right]$ cation. These investigators characterized nanoparticle formation as well as aggregation effects on emission spectra, noting that an exci-aggremer peak was observed in the nanoGUMBOS. They determined that the appearance of this exci-aggremer peak may potentially be useful for light-harvesting applications [16]. In order to showcase this potential application, these investigators also explored the initial characterization for their nanoGUMBOS system as a potential Forster Resonance Energy Transfer (FRET) donor. Using the hydrophobic acceptor dye 4-(Dicyanomethylene)2-methyl-6-(4-dimethylaminostyryl)-4H pyran (DCM), these investigators synthesized nanoGUMBOS with DCM to investigate FRET efficiency for potential applications as energy relay dyes in DSSCs.

More recently, Azevedo [17] and coworkers have investigated automation-based toxicity assays to evaluate rapid processes for determining toxicity of ionic compounds with yeast viability [17]. In this work, these investigators employed spectrophotometric methods to determine toxicity of ionic materials: eleven ILs, three Deep Eutectic Solvents (DES), and three selected erythrosine B-based GUMBOS (EB-GUMBOS). Among EB-GUMBOS, cations exchange was employed to more hydrophobic, water insoluble systems using trimethylhexadecylammonium ([N11116]), tetrabutylphosphonium ([P4444]), and 16BAC. Automation procedures for sequential injection analyses based on loss of cell viability as determined by methylene blue dye reduction capability of yeast cells (Saccharomyces cerevisiae) were developed. While these investigators confirmed that all ionic materials studied resulted in loss of $S$. cerevisiae cell viability (GUMBOS > ILs > DES), they also confirmed that viability loss was dependent on the dose of molecules employed. Among the three different ionic groups studied in EB-GUMBOS, analysis of results from this method indicated an increasing range in toxicity from [N11116] $>$ [P4444] $>$ 16BAC EB-GUMBOS. Although hydrophobicity was not directly measured, this report highlights the potential tunable effects from cation-exchange on possible antimicrobial development for EBGUMBOS [17].

\section{Effects of anion-exchange in GUMBOS and related self- assembly}

Counter anion exchange has been employed to study a variety of phenomena in the field of fluorescent ionic materials [18]. In one application for optoelectronics, anion variation with carbazolebased GUMBOS for highly sought after blue Organic Light Emitting Diodes (OLEDs) was reported [4]. Based on the requisite amorphous nature of blue emissive layers, a targeted carbazole cation was developed. In this work, three anions were employed for carbazoleGUMBOS syntheses, i.e. triflate ([OTf]), [NTf2], and [BETI] anions. Comparative characterization studies were performed to examine and achieve stable blue emission in the solid-state through uniform production of GUMBOS films using a simple electrospray procedure. GUMBOS containing the [BETI] anion were determined to have the highest photostability over six hours. In this work, these investigators demonstrated the successful production of emissive films with amorphous character by varying hydrophobic anions. Moreover, this work highlights the use of simple synthetic strategies for functional small ionic molecules as potential blue solid-state OLED emissive layers.

Anion exchange effects on the self-assembly and resultant fluorescence properties of GUMBOS based on the cationic cyanine dye 1,10,3,3,30,30-hexamethylindotricarbocyanine, or ([HMT]) has also been explored [19]. By using a simple reprecipitation technique, these investigators observed counterion-based variations in nanoparticle size and aggregation dynamics. For example, spherical nanoGUMBOS sizes ranged from 30 to $100 \mathrm{~nm}$ in diameter, and J-aggregation was determined to be the dominant form of assembly in [HMT][AOT], whereas H-aggregation was prevalent in [HMT][TFP4B]. Another area for nanoGUMBOS evaluation is for potential use as agents for imaging and anticancer therapies. In this regard, Bwambok and coworkers investigated near infraredbased GUMBOS as in vitro fluorescent contrast imaging agents using nanoGUMBOS. This study assessed nanoGUMBOS stability and evaluation of dye leakage within cellular systems. Using [HMT] [AOT] GUMBOS, a homogenous distribution of nanoGUMBOS with an average diameter size of $71 \pm 16 \mathrm{~nm}$ in the cytoplasm was observed with minimal dye leakage.

In 2013, Magut [20] and coworkers investigated anion exchange effects on the rhodamine 6G ([R6G]) cation [20]. While the parent chloride salt of [R6G] was previously reported as toxic to both cancer and normal cells, more hydrophobic anions [R6G][BETI] and [R6G][TPB] in the form of nanoparticles yielded lower toxicity to normal cell lines and were determined to inhibit proliferation of 
cancer cells at low concentrations. In contrast, sodium and lithium anions were determined to be non-toxic to both cell lines in in vitro studies. Since this time, further exploration into templating these nanoGUMBOS with cyclodextrins and evaluation of their resultant cellular uptake and in vitro efficacy [11], as well as antitumor efficacy of GUMBOS derived from other rhodamine cation derivatives have also been explored [12]. Studies from these researchers have shown that nanoparticle formation is key to the observed selective toxicity [11]. Several reports of anion exchange with tunable antitumor and imaging activities have also been reported [3,21-24].

\section{Magnetic components in task-specific ionic materials}

Magnetic materials, and nanomaterials, have been a target for studying applications in magnetic resonance imaging [25,26], solid phase preconcentration and extraction [27], and drug delivery [28]. In many such studies, multi-step syntheses are required to achieve task-specific magnetic components. In this respect, magnetic ionic materials provide a simple approach to design and evaluation of task-specific GUMBOS with multifunctionality. As an example, Tesfai [26] and coworkers explored magnetic anion effect on nanoGUMBOS formation with nonmagnetic [BMIm][BF4] and magnetic [BMIm]
[FeCl4] [26]. In this report, these investigators employed an AOTsurfactant templating method with in situ GUMBOS synthesis (Figure 2a). Using non-magnetic [BMIm][BF4], these investigators determined that nanoparticle size and distribution was easily controlled, and a range of concentration-dependent particle sizes were measured from $14.7 \mathrm{~nm} \pm 2.2 \mathrm{~nm}$ to $68.0 \mathrm{~nm} \pm 17.0 \mathrm{~nm}$ with $0.2 \mathrm{M}$ to $0.6 \mathrm{M}$ reagent concentrations, respectively. NanoGUMBOS from [BMIm] $[\mathrm{FeCl} 4]$ were observed to be larger in particle size ranging from $98 \mathrm{~nm} \pm 17 \mathrm{~nm}$ to $199 \mathrm{~nm} \pm 26 \mathrm{~nm}$ with 0.3 and $0.4 \mathrm{M}$ reagent concentrations, respectively. Overall, these investigators demonstrated the soft matter properties of these magnetic nanoGUMBOS. Room temperature ILs have been employed as cationic agents using hydrophobic counterions for phenolic compound remediation [29]. These authors followed a traditional strategy for magnetic IL synthesis, using [P66614][Cl] and $\mathrm{FeCl}_{3}$ to form [P66614][FeCl4] (Figure 2b). After comparative extraction analyses to non-magnetic ILs and evaluating $\mathrm{pH}$ dependence on extraction efficacy, these investigators determined that [P66614] [FeCl4] exhibited a significantly higher extraction capacity for phenolic compounds, including extraction of a polychlorinated phenol from soil of a Superfund site. a)

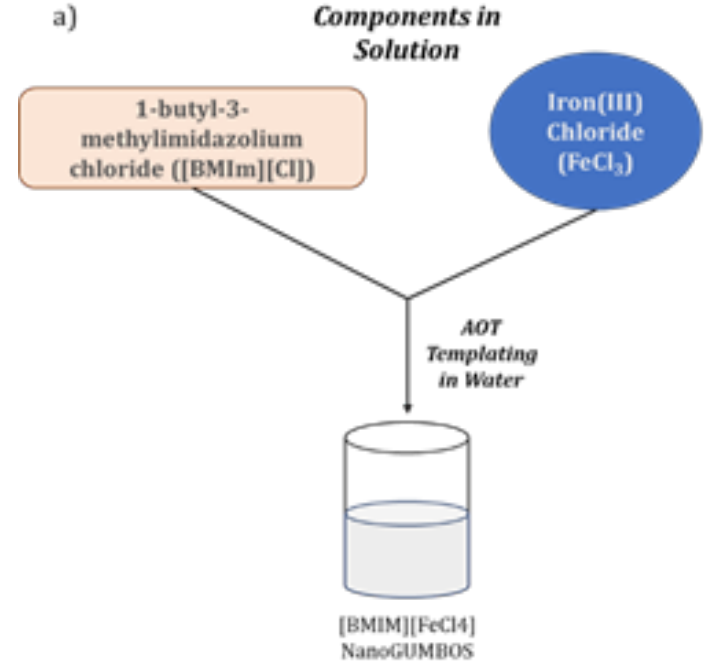

b)

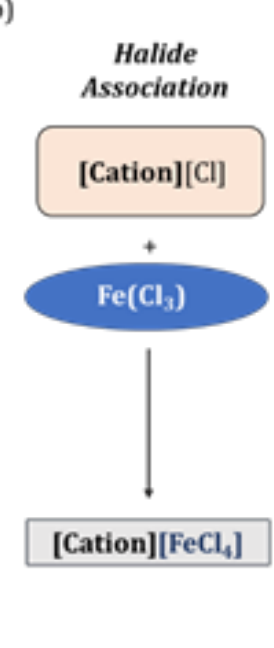

c)

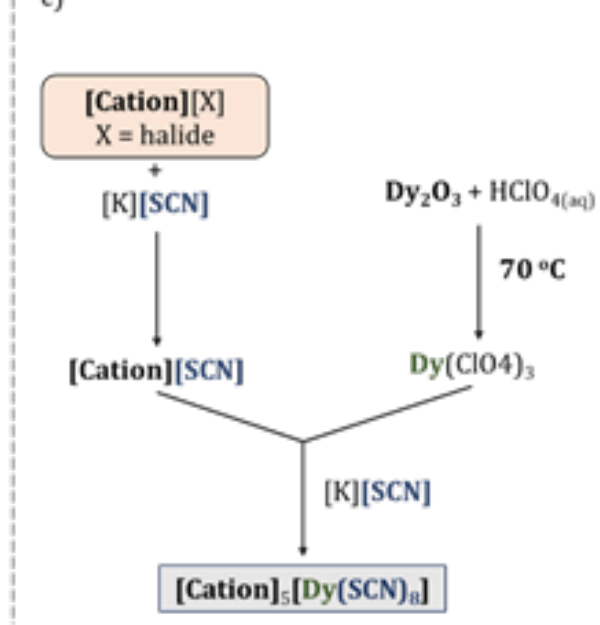

Figure 2: Schematic of magnetic synthesis strategies for a) in situ magnetic nanoGUMBOS, b) halide association, and c) dysprosium-based GUMBOS.

Chiral-magnetic ionic liquids synthesized from methyl esters of amino acids and iron(III) chloride $\left(\mathrm{FeCl}_{3}\right)$ have also been explored [30]. In one study, five cationic amino-acid methyl esters were employed for synthesis, and the resultant magnetic, chiral ILs were characterized and evaluated for potential enantiomeric recognition ability of these magnetic materials. Although these materials presented as liquids at room temperature, an interesting result was observed in which these magnetic, chiral ILs displayed a phase transition at low temperatures from the liquid to a glassy state [30]. As a result of their unique liquid-crystalline characteristics, a highly ordered, multi-functional ionic material was developed based on $(1 R, 2 S)-(-)-N-M e t h y l e p h e d r i n e$, dysprosium (a luminescent lanthanide metal with the highest reported magnetic moment), and thiocyanate [31]. Although these materials exhibited relatively low melting points, solid crystalline phases were observed at room temperature. Chiral, luminescent, and magnetic properties were characterized, and transitions from solid to liquid crystalline states were also synthesized using a similar scheme as shown in Figure 2c. Thermal studies to explore liquid crystalline ordering were performed, as well as luminescent properties, to explore and investigate phase dependence on these properties.

Further studies regarding magnetic extraction have been recently reported. In 2021, Berton and coworkers also explored quantification and remediation of estrogen compounds by varying magnetic components using [P66614] cations with anions of increasing hydrophobicity [FeCl4], [Fe(CN)6], and [Dy(SCN)8]. 
Optimal conditions were determined and ILs with [Fe(CN)6], and $[\mathrm{Dy}(\mathrm{SCN}) 8]$ were determined to have the highest extraction efficiencies [32]. Cong [23] and coworkers have also explored hemoglobin $(\mathrm{Hb})$ isolation using the cation 1-hexadecyl-3-methylimidazolium with [Dy(SCN)8] anion to form task-specific magnetic nanoGUMBOS. Size optimization, magnetic moment, and selective extraction efficiency for $\mathrm{Hb}$ were reported. This work highlights the potential utility of these easily synthesized nanoGUMBOS, since $\mathrm{Hb}$ isolation from whole blood was also presented. Based on these reports of hydrophobicity and selective extraction, further exploration of magnetic, GUMBOS is warranted to explore additional task-specific material development. Thus, these recent developments highlight the need to continue investigation of counterion tuning strategies to evaluate the potential multi-faceted capabilities of these task-specific GUMBOS and nanoGUMBOS.

\section{Conclusion}

In general, the variability of ionic materials holds promise for a wide array of applications. While scientific development has largely focused on use of ionic liquids for material development, this review has detailed several advantages that accrue from use of a group of uniform materials based on organic salts (GUMBOS). Our group, along with several other research groups have focused on the broad scope and evaluation of these solid-state IL-analogues. The simplicity of syntheses of these ionic systems allow researchers to focus on rapid development and application of these materials in a relatively low-cost and labor efficient approach. Moreover, targeting task-specific and potentially multi-functional GUMBOS provides new paths for investigation and exploration of easily synthesized ionic materials for applications in many different fields of study.

\section{Acknowledgement}

The authors gratefully acknowledge financial support through NASA cooperative agreement NNX 16AQ93A under contract number NASA/LEQSF (2016-2019)-Phase 3-10, and the National Science Foundation under Grant Nos. CHE-1905105 and HRD-1736136. Any opinions, findings, and conclusions or recommendations expressed in this material are those of the author(s) and do not necessarily reflect the views of the National Science Foundation.

\section{Conflicts of Interest}

The authors declare no conflict of interest.

\section{References}

1. Warner IM, Zahab EB, Siraj N (2014) Perspectives on moving ionic liquid chemistry into the solid phase. Analytical Chemistry 86(15): 7184-7191.

2. Kaur P, Chopra HK (2020) Recent progress in synthesis and applications of tunable materials and nanomaterials based on organic salts. Chemistry Select 5(42): 13033-13053.

3. Azevedo AMO, Santos JLM, Warner IM, Saraiva MLMFS (2020) GUMBOS and nano GUMBOS in chemical and biological analysis: A review. Analytica Chimica Acta 1133: 180-198.

4. Siraj N, Hasan F, Das S, Kiruri LW, Gall SKE, et al. (2014) Carbazolederived group of uniform materials based on organic salts: Solid state fluorescent analogues of ionic liquids for potential applications in organic-based blue light-emitting diodes. The Journal of Physical Chemistry C 118(5): 2312-2320.

5. Galpothdeniya WIS, Regmi BP, McCarter KS, Rooy DSL, Siraj N, et al. (2015) Virtual colorimetric sensor array: Single ionic liquid for solvent discrimination. Analytical Chemistry 87(8): 4464-4471.

6. Pérez RL, Ayala CE, Park JY, Choi JW, Warner IM (2021) Coating-based quartz crystal microbalance detection methods of environmentally relevant volatile organic compounds. Chemosensors 9(7): 153.

7. Pérez RL, Ayala C, Warner I (2021) In group of uniform materials based on organic salts (GUMBOS): A review of their solid-state properties and applications.

8. Lopez KM, Hobden JA, Warner IM (2020) Octenidine/carbenicillin GUMBOS as potential treatment for oropharyngeal gonorrhoea. Journal of Antimicrobial Chemotherapy 75(12): 3576-3581.

9. Cole MR, Hobden JA, Warner IM (2015) Recycling antibiotics into GUMBOS: A new combination strategy to combat multi-drug-resistant bacteria. Molecules 20(4): 6466-6487.

10. Cole MR, Li M, Jadeja R, Zahab EB, Hayes D, et al. (2013) Minimizing human infection from Escherichia coli 0157: H7 using GUMBOS. Journal of Antimicrobial Chemotherapy 68(6): 1312-1318.

11. Bhattarai N, Mathis JM, Chen M, Pérez RL, Siraj N, et al. (2018) Endocytic selective toxicity of rhodamine 6G nano GUMBOS in breast cancer cells. Molecular Pharmaceutics 15(9): 3837-3845.

12. Bhattarai N, Chen M, Pérez LR, Ravula S, Strongin RM, et al. (2020) Comparison of chemotherapeutic activities of rhodamine-based GUMBOS and nano GUMBOS. Molecules 25(14): pp. 3272.

13. Pramanik P, Sahoo R, Das KS, Halder M (2020) Fabrication of a GUMBOSbased acid-base indicator: Smart probe for sensing acids and bases in any solvent. Physical Chemistry Chemical Physics 22(48): 28045-28054.

14. Bwambok DK, Siraj N, Macchi S, Larm NE, Baker GA, et al. (2020) QCM Sensor arrays, electroanalytical techniques and NIR spectroscopy coupled to multivariate analysis for quality assessment of food products, raw materials, ingredients and foodborne pathogen detection: Challenges and Breakthroughs. Sensors 20(23): 6982.

15. Kolic PE, Siraj N, Hamdan S, Regmi BP, Warner IM (2016) Synthesis and characterization of porphyrin-based GUMBOS and nano GUMBOS as improved photosensitizers. The Journal of Physical Chemistry C 120(9): $5155-5163$

16. Pramanik P, Das SK, Halder M (2020) FRET-selective and ion-exchange responsive smart nano GUMBOS from functionalized pyrene: First observation of excited state aggregation (Exciaggremer) inside crystalline nanoball. The Journal of Physical Chemistry C 124(8): 47914801.

17. Azevedo AMO, Vilaranda AG, Neves AFDC, Sousa MJ, Santos JLM, et al. (2021) Development of an automated yeast-based spectrophotometric method for toxicity screening: Application to ionic liquids, GUMBOS, and deep eutectic solvents. Chemosphere 277: 130227.

18. Siraj N, El Zahab B, Hamdan S, Karam TE, Haber LH, et al. (2016) Fluorescence, Phosphorescence, and Chemiluminescence. Analytical Chemistry 88(1): 170-202.

19. Das S, Bwambok D, El Zahab B, Monk J, de Rooy SL, et al. (2010) Nontemplated approach to tuning the spectral properties of cyaninebased fluorescent NanoGUMBOS. Langmuir 26(15): 12867-12876.

20. Magut PKS, Das S, Fernand VE, Losso J, McDonough K, et al. (2013) Tunable cytotoxicity of rhodamine $6 \mathrm{G}$ via anion variations. Journal of the American Chemical Society 135(42): 15873-15879.

21. Huang Y, Zhang G, Hu F, Jin Y, Zhao R, et al. (2016) Emissive nanoparticles from pyridinium-substituted tetraphenylethylene salts: imaging and selective cytotoxicity towards cancer cells in vitro and in vivo by varying counter anions. Chemical Science 7(12): 7013-7019. 
22. Huang Y, You X, Wang L, Zhang G, Gui S, et al. (2020) Pyridiniumsubstituted tetraphenylethylenes functionalized with alkyl chains as autophagy modulators for cancer therapy. Angewandte Chemie International Edition 59(25): 10042-10051.

23. Chen M, Bhattarai N, Cong M, Pérez RL, McDonough KC, et al. (2018) Mitochondria targeting IR780-based nanoGUMBOS for enhanced selective toxicity towards cancer cells. RSC Advances 8(55): 3170031709.

24. Chen M, Pérez RL, Du P, Bhattarai N, McDonough KC, et al. (2019) Tumortargeting NIRF NanoGUMBOS with cyclodextrin-enhanced chemo/ photothermal antitumor activities. ACS Applied Materials \& Interfaces 11(31): 27548-27557.

25. Kudr J, Haddad Y, Richtera L, Heger Z, Cernak M, et al. (2017) Magnetic nanoparticles: From design and synthesis to real world applications. Nanomaterials 7(9): 243.

26. Tesfai A, El Zahab B, Kelley AT, Li M, Garno JC, et al. (2009) Magnetic and nonmagnetic nanoparticles from a group of uniform materials based on organic salts. ACS Nano 3(10): 3244-3250.

27. Chen L, Wang T, Tong J (2011) Application of derivatized magnetic materials to the separation and the preconcentration of pollutants in water samples. TrAC Trends in Analytical Chemistry 30(7): 1095-1108.
28. Liao J, Huang H (2020) Review on magnetic natural polymer constructed hydrogels as vehicles for drug delivery. Biomacromolecules 21(7): 25742594.

29. Deng N, Li M, Zhao L, Lu C, de Rooy SL, et al. (2011) Highly efficient extraction of phenolic compounds by use of magnetic room temperature ionic liquids for environmental remediation. Journal of Hazardous Materials 192(3): 1350-1357.

30. Li M, De Rooy SL, Bwambok DK, El Zahab B, Ditusa JF, et al. (2009) Magnetic chiral ionic liquids derived from amino acids. Chemical Communications (45): 6922-6924.

31. Lu C, Das S, Siraj N, Magut PKS, Li M, et al. (2015) Spectral and physicochemical characterization of dysprosium-based multifunctional ionic liquid crystals. The Journal of Physical Chemistry A 119(20): 47804786.

32. Berton P, Siraj N, Das S, de Rooy S, Wuilloud RG, et al. (2021) Efficient low-cost procedure for microextraction of estrogen from environmental water using magnetic ionic liquids. Molecules 26(1): 32. 